\title{
A discrete time domain approach on time delay estimation
}

\author{
Jorma Kekalainen
}

\section{ABSTRACT}

A time delay estimation method based on the discrete time domain approach is introduced here. In this dual-channel time delay estimation model, the criterion function compares the time differences of time sequences between channels, not the magnitude values of time functions as in the conventional cross-correlation method. An estimation task is formulated as an extreme value problem in discrete index space. Using the index delay giving extreme value to the criterion function, it is possible to find the best estimate for time delay distribution in the meaning of that criterion. Using this method, the estimated delay distribution and criterion function are clearly separated. Thus, there are no theoretical problems in the determination of the average time delay or velocity in the non-constant or changing time delay case as long as a sufficient statistical similarity (correlation) exists between channel signals.

The theoretical values of several criterion functions and the probability of occurrence of an anomalous estimate with the crosscovariance criterion function are derived. A basic performance analysis of the estimation method is presented. Some potential real-time supervision methods based on the use of criterion functions in the detection of the possible unreliability of the time delay estimate are outlined.

\section{Section: RESEARCH PAPER}

Keywords: Time delay estimation; criterion function; index delay; cross-correlation; reliability checking

Citation: Jorma Kekalainen, A discrete time domain approach on time delay estimation, Acta IMEKO, vol. 8, no. 1, article 11, March 2019, identifier: IMEKOACTA-08 (2019)-01-11

Section Editor: Konrad Jedrzejewski, Warsaw University of Technology, Poland

Received April 26, 2018; In final form February 19, 2019; Published March 2019

Copyright: @ 2019 IMEKO. This is an open-access article distributed under the terms of the Creative Commons Attribution 3.0 License, which permits unrestricted use, distribution, and reproduction in any medium, provided the original author and source are credited.

Corresponding author: Jorma Kekalainen, e-mail: jormakekalainen@outlook.com

\section{INTRODUCTION}

Time delay estimation plays a significant role in numerous engineering applications, specifically signal processing [1], [2]. The problem of estimating time delays between noisy signals has attracted considerable attention over the years due to the variety of applications in areas such as acoustics, sonar, radar, flow, and velocity measurements. The accurate estimation of time delay is often complicated by conditions of poor signal-to-noise ratio, a changing time delay, multipath propagation, and dispersion. Various time delay estimation procedures have been proposed and implemented over the decades. The general goal has been to minimise the variance of the time delay estimates in the presence of uncorrelated noise at the receiving ends.

For time delay estimation in linear systems, there are three basic operations involved: 1) filtration of input signals in the case of non-white signal and noise spectral characteristics in order to accentuate the frequency bands with good signal-to-noise ratio; 2) comparison (or correlation) of the two filtered waveforms in order to apply the detection by means of threshold; and 3) computation of time delay estimate.

Time delay estimation procedures based on cross correlation, smoothed coherence transforms, unit impulse response, and maximum likelihood estimates can generally be viewed as the inverse Fourier transforms of normalised (weighted) cross- spectral density functions that yield delay estimates in time domain terms. Many of the methods proposed for time delay estimation have been shown to be related by means of the generalised cross-correlation (GCC) approach, which involves filtering the received signals and estimating the time delay as the time lag where the cross-correlation function of filtered signals is at its maximum [3].

The GCC function and the estimate of time delay can be expressed in the well-known form

$\left\{\begin{array}{l}R(\tau)=\mathrm{F}^{-1}\left\{W(f) G_{12}(f)\right\} \\ \tau_{\text {Delay }}=\operatorname{Arg}\left\{\operatorname{Max}_{\tau}[R(\tau)]\right\}\end{array}\right.$

where $\mathrm{F}^{-1}$ is the inverse Fourier transform, $W(f)=H_{1}(f) H_{2}(f)^{*}$ is a weighting function determined by the filter frequency responses (* is used to denote the complex conjugate), and $G_{12}$ is the cross power density spectrum of the received sensor signals 1 and 2. The Arg symbol denotes an argument operator, that picks the time lag or delay argument $\tau$ of $R$.

Cross-correlation is mainly suggested in applications in which some knowledge of time delays is required, such as the velocity measurement of solid or non-solid material flow (e.g. steel and paper strips; road and rail vehicles; and mixtures of solids, liquids, and gas) and range measurement by sonar and radar [4]-[10]. 


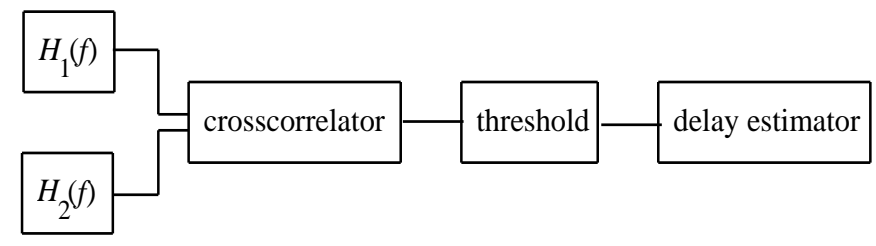

Figure 1. Block diagram model for dual-channel delay estimation with the generalised correlation method.

If the thresholding device in the basic model of Figure 1 is moved to the front of the cross-correlator, a simple correlation scheme is obtained [11]. Threshold crossing can be used as a criterion to save data, which is then processed by correlation and delay estimation. This algorithm, which allows for fast polarity correlation, was proposed by Henry [12].

Henry's idea is based on the fact that information used by a polarity cross-correlator comprises the actual zero-crossing times of the signals [13]. Henry noted that the zero crossings are randomly distributed in time; hence, the observation of these zero crossings gives data with a sufficient time resolution without the need for sampling the data at close intervals that were previously thought necessary to give an adequate resolution.

If polarity information is replaced by crossing over times, then the time delay estimate is the time interval between the corresponding crossing over times on different channels. To find the crossing over times corresponding to each other in both channels, some criterion function is needed for the decisionmaking process involved in the detection of the time delay estimate. The aim of this paper is to present the basic idea and analysis of a method that utilises pre-processed time signals and some simple criterion functions in time delay estimation.

\section{TIME DIFFERENCE DISTRIBUTION METHOD}

\subsection{Time differences and criterion functions}

To determine an estimate for the time delay between two axially separated sensors, time differences are formed using the same sensor arrangement as in conventional cross-correlation methods, and the utilisation of information included in the zerocrossing times of the signals or any time moment is actually based on some other detectable and randomly distributed characteristics (vortices in turbulent flow, gas bubbles, clumps of particles, macroscopic objects, surface patterns, injected chemical or radioisotope markers, etc.) in flow. As a result, two primary time sequences $\{t\}$ and $\{T\}$ are obtained from one channel (input) and from the other channel (output). Of course, in any case, a sufficient statistical similarity (correlation) between channel signals with some time delay is an essential prerequisite for the successful utilisation of the method.

The time difference distribution (TDD) method for solving time delay estimation problems is based on the use of any criterion function that is able to utilise that statistical similarity between the channel signals and to fix the time elements corresponding each other in sequences $\{t\}$ and $\{T\}$. Using the index delay that gives the extreme value (maximum or minimum value depending on the criterion type) to the criterion function, it is possible to select one collection of $(t, T)$-pairs and to calculate the best estimate for time delay distribution $\left(\tau_{j}, j=1, \ldots\right)$ within the meaning of that criterion. With the help of estimated time delay distribution, the velocity distribution $\left(L / \tau_{j}, j=1, \ldots\right)$ can be calculated when the distance $(L)$ between the axially separated sensors is known. Of course, sufficient statistical similarity (correlation) between channel signals sets an upper limit for the distance $L$.

All suitable criterion functions with the previous time difference formulation of the time delay estimation problem have been inherently defined in the discrete (index) domain. Next, mainly as an example, we present the simplest possible criterion functions. The theoretical counterparts of these functions are presented in section 2.2.

The first criterion function is called the sample variance delay function $\hat{e}_{v d}$. As a criterion of the best estimate, we use the minimum value of the sample variance delay function

$\operatorname{Min}_{k}\left\{\hat{e}_{v d}(k)\right\}=\operatorname{Min}_{k}\left\{\left\langle\Delta^{2}(i+k, i)\right\rangle-\langle\Delta(i+k, i)\rangle^{2}\right\}$

where $\Delta(i+k, i)=T(i+k)-t(i), T(i+k)$ is the $(i+k)$ th element of $\{T\}, t(i)$ is the $i$ th element of $\{t\}, k$ is an index delay, and $\langle\cdot\rangle$ denotes a sample mean.

The second criterion function is called the sample average delay difference function $\hat{e}_{a d d}$

$$
\begin{aligned}
& \operatorname{Min}_{k}\left\{\hat{e}_{\text {add }}(k)\right\} \\
& \quad=\operatorname{Min}_{k}\{\langle|\Delta(i+k, i)-\Delta(i+k-1, i-1)|\rangle\}
\end{aligned}
$$

where $|\cdot|$ is the absolute value.

The third criterion function is the sample variance delay difference function $\hat{e}_{v d d}$

$$
\begin{aligned}
& \operatorname{Min}_{k}\left\{\hat{e}_{v d d}(k)\right\} \\
&=\operatorname{Min}_{k}\left\{\begin{array}{c}
\left\langle|\Delta(i+k, i)-\Delta(i+k-1, i-1)|^{2}\right\rangle \\
-\langle|\Delta(i+k, i)-\Delta(i+k-1, i-1)|\rangle^{2}
\end{array}\right\} .
\end{aligned}
$$

The fourth criterion function is correlation coefficient $\hat{e}_{c o r}$

$$
\begin{aligned}
& \operatorname{Max}_{k}\left\{\hat{e}_{c o r}(k)\right\} \\
&=\operatorname{Max}_{k}\left\{\frac{\langle\Delta T(i+k) \Delta t(i)\rangle-\langle\Delta T(i+k)\rangle\langle\Delta t(i)\rangle}{\sqrt{\operatorname{Vâr}\{\Delta T\} \operatorname{Var}\{\Delta t\}}}\right\}
\end{aligned}
$$

where

$$
\begin{aligned}
& V \hat{a} r\{\Delta T\}=\left\langle\Delta T^{2}(i+k)\right\rangle-\langle\Delta T(i+k)\rangle^{2} \\
& V \hat{a} r\{\Delta t\}=\left\langle\Delta t^{2}(i)\right\rangle-\langle\Delta t(i)\rangle^{2} \\
& \Delta T(i+k)=T(i+k)-T(i+k-1) \\
& \Delta t(i)=t(i)-t(i-1) .
\end{aligned}
$$

As the denominator and product of averages $\langle\Delta T(i+k)\rangle$ and $\langle\Delta t(i)\rangle$ are constants independent of index delay $k$ in stationary sequences, the maximisation of the correlation coefficient is equivalent to the maximisation of the crosscorrelation function

$$
\operatorname{Max}_{k}\left\{\hat{e}_{c c}(k)\right\}=\operatorname{Max}_{k}\{\langle\Delta T(i+k) \Delta t(i)\rangle\}
$$

where $\hat{e}_{c c}(k)$ is sample cross-correlation function of time intervals.

The minimisation of the quadratic delay difference function

$\operatorname{Min}_{k}\left\{\hat{e}_{q d d}(k)\right\}=\operatorname{Min}_{k}\left\{\left\langle|\Delta T(i+k)-\Delta t(i)|^{2}\right\rangle\right\}$

in stationary sequences is also equivalent to the maximisation of the correlation coefficient and the cross-correlation function.

\subsection{Theoretical values of some criterion functions}

In the following, stationary FIFO is assumed, meaning here that the detectable objects in the flow do not mix in the space between the sensors $\Rightarrow$ undistorted delay distribution case. In fluid mechanics, a plug flow is a simple model of the velocity 
profile of a fluid flowing in a pipe. In plug flow, the velocity of the fluid is assumed to be constant across any cross-section of the pipe perpendicular to the axis of the pipe. In an ideal plug flow, the fluid is not mixed in the axial direction in the space between the sensors, and any infinitesimal fluid plug element that enters the space between sensors at time $t$ exits the space between the sensors at time $T=t+\tau$, where $\tau$ is the delay time of any plug between the sensors. The delay time distribution function is, therefore, the Dirac delta function at $\tau$. However, any real plug flow has a delay time distribution that is a 'narrow' pulse around the mean delay time. Plug flow is a special type of previously assumed FIFO flow.

The expectation of the sample variance delay function $\hat{e}_{v d}$ (in the stationary FIFO case) for independent random variables $\Delta t$ and $\tau$ can be presented in the form

$$
\begin{aligned}
e_{v d}(k) & =E\left\{\hat{e}_{v d}\right\}=E\left\{\left\langle\Delta^{2}(i+k, i)\right\rangle-\langle\Delta(i+k, i)\rangle^{2}\right\} \\
& =\left|k-k_{0}\right| \operatorname{Var}\{\Delta t\}+\operatorname{Var}\{\tau\}
\end{aligned}
$$

where $E$ is the expectation operator, $\operatorname{Var}\{\Delta t\}$ is the variance of the time interval in the sequence $\{t\}, \operatorname{Var}\{\tau\}$ is the variance of time delay $\tau$, and $k_{0}$ is a fixed index delay that minimises the criterion function $\hat{e}_{v d}$.

There is a minimum of $e_{v d}$

$\underset{k=k_{0}}{\operatorname{Min}}\left\{e_{v d}(k)\right\}=\operatorname{Var}\{\tau\}$.

Therefore, moving $\left(k_{-}-k_{0}\right)$ index units from the optimum index delay $k_{0}$, the value of the criterion function increases by an amount of time interval variance weighted with the absolute value of $\left(k-k_{0}\right)$ index units, as we see in Figure 2.

The sample criterion function previously denoted as $\hat{e}_{a d d}$ can be presented in the form

$\hat{e}_{\text {add }}(k)$

$$
\begin{aligned}
& =\langle| \Delta \tau\left(\left|k-k_{0}\right|+i\right)+\Delta t\left(\left|k-k_{0}\right|+i\right) \\
& -\Delta t(i)|\rangle \geq\left\langle\left|\Delta \tau\left(\left|k-k_{0}\right|+i\right)\right|\right\rangle
\end{aligned}
$$

where the time delay difference is $\Delta \tau(s)=\tau(s)-\tau(s-1)$.

Equality in equation (10) is true if time interval $\Delta t(\cdot)$ between the sequential elements in sequence $\{t\}$ is constant or index delay $k=k_{0}$, which minimises the value of criterion function $\hat{e}_{a d d}$.

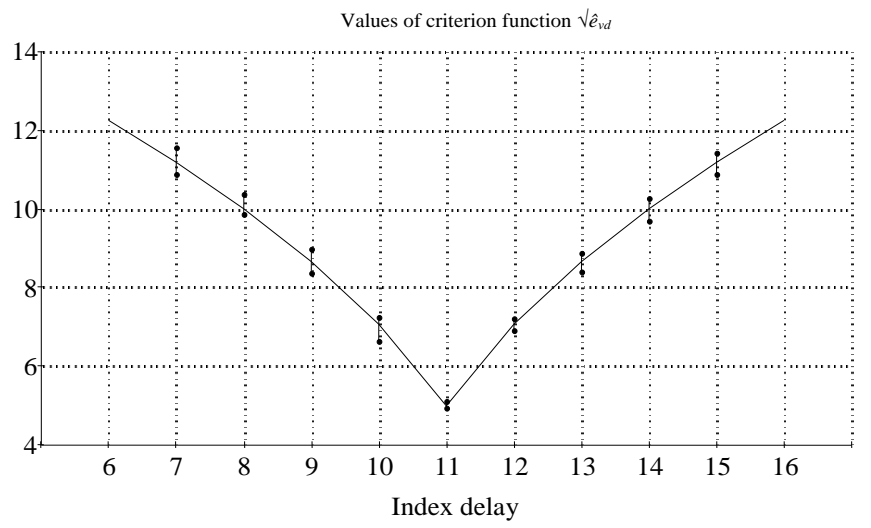

Figure 2. Theoretical and sample values of the square root of the variance delay function $\hat{e}_{v d}$. All 11 theoretical values presented in this index delay window are combined with the line. Sample distributions in the simulation experiments are approximately Gaussian $\Delta t \sim N\left(30,5^{2}\right)$ and $\tau \sim N\left(300,5^{2}\right)$. The sample size is 1000 . Theoretical distributions are exactly Gaussian with the previous parameters. The bar denotes the variation range of the sample values in 30 experiments.
The theoretical value of $\hat{e}_{a d d}(k)$ is

$e_{a d d}(k)=\int_{-\infty}^{\infty}|z| \int_{-\infty}^{\infty} f_{\Delta[\Delta t]}(z-\Delta \tau) f_{\Delta \tau}(\Delta \tau) d \Delta \tau d z$

where

$f_{\Delta[\Delta t]}(z-\Delta \tau)=\int_{-\infty}^{\infty} f_{\Delta t}(\Delta t) f_{\Delta t}(z-\Delta \tau-\Delta t) d \Delta t$,

$f_{\Delta \tau}(\Delta \tau)=\int_{-\infty}^{\infty} f_{\tau}(\tau) f_{\tau}(\Delta \tau-\tau) d \tau$,

$z=\Delta[\Delta t]+\Delta \tau$

$f_{\Delta t}$ is a probability density function of $\Delta t$, and $f_{\tau}$ is a probability density function of $\tau$.

The minimum value of $e_{\text {add }}$ is

$\operatorname{Min}_{k=k_{0}}\left\{e_{\text {add }}(k)\right\}=\int_{-\infty}^{\infty}|z| \int_{-\infty}^{\infty} f_{\tau}(\tau) f_{\tau}(z-\tau) d \tau d z$

where $z=\Delta \tau$.

Figure 3 shows the coincidence of the theoretical and sample values of the criterion function $\hat{e}_{\text {add }}$.

The sample variance delay difference function $\hat{e}_{v d d}$ can be presented in the form

$\hat{e}_{v d d}(k)$

$=\left\langle\left|\Delta \tau\left(\left|k-k_{0}\right|+i\right)+\Delta t\left(\left|k-k_{0}\right|+i\right)-\Delta t(i)\right|^{2}\right\rangle$

$-\left\langle\left|\Delta \tau\left(\left|k-k_{0}\right|+i\right)+\Delta t\left(\left|k-k_{0}\right|+i\right)-\Delta t(i)\right|\right\rangle^{2}$.

The theoretic counterpart of $\hat{e}_{v d d}$ with the previous notes is

$e_{v d d}(k)$

$$
\begin{aligned}
& =\left[\int_{-\infty}^{\infty} z^{2} \int_{-\infty}^{\infty} f_{\Delta[\Delta t]}(z-\Delta \tau) f_{\Delta \tau}(\Delta \tau) d \Delta \tau d z\right] \\
& -\left[\int_{-\infty}^{\infty}|z| \int_{-\infty}^{\infty} f_{\Delta[\Delta t]}(z-\Delta \tau) f_{\Delta \tau}(\Delta \tau) d \Delta \tau d z\right]^{2} .
\end{aligned}
$$

The minimum value of $e_{v d d}$ is

$$
\begin{aligned}
\operatorname{Min}_{k=k_{0}}\left\{e_{v d d}(k)\right\} & \\
= & {\left[\int_{-\infty}^{\infty} z^{2} \int_{-\infty}^{\infty} f_{z}(\tau) f_{\tau}(z-\tau) d \tau d z\right] } \\
& -\left[\int_{-\infty}^{\infty}|z| \int_{-\infty}^{\infty} f_{z}(\tau) f_{\tau}(z-\tau) d \tau d z\right]^{2} .
\end{aligned}
$$

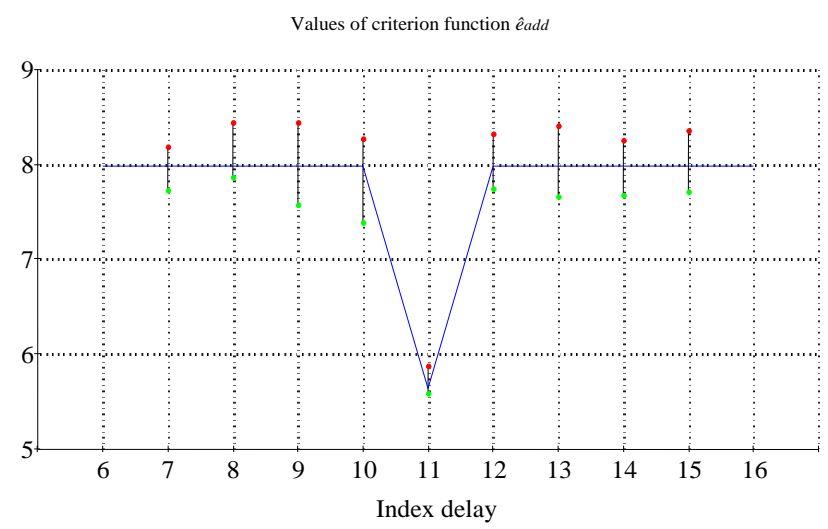

Figure 3. Theoretical and sample values of the average delay difference function $\hat{e}_{\text {add }}$ with the same statistics as in Figure 2 . Theoretical values computed from the equation (11) and equation (12) are combined with the line. The bar denotes the variation range of the sample values in 30 experiments. 
Figure 4 shows the coincidence of the theoretical and sample values of the criterion function.

The theoretical counterpart of the correlation coefficient can be presented in the form of normalised cross-covariance function

$e_{c o r}(k)=\frac{\operatorname{Cov}\{\Delta T(i+k), \Delta t(i)\}}{\sqrt{\operatorname{Var}\{\Delta T(i+k)\} \operatorname{Var}\{\Delta t(i)\}}}$.

The maximum value of $e_{c o r}$ is

$\operatorname{Max}_{k=k_{0}}\left\{e_{c o r}(k)\right\}=\sqrt{\frac{\operatorname{Var}\{\Delta t\}}{\operatorname{Var}\{\Delta t\}+2 \operatorname{Var}\{\tau\}}}$

if $\Delta t$ and $\tau$ are independent.

Equivalently. cross-correlation function can be presented in the form

$e_{c c}(k)=R_{\Delta t \Delta t}(k)+R_{\Delta t \Delta \tau}(k)$

where $R_{\Delta_{t} \Delta_{t}}$ is the autocorrelation function of $\Delta t$, and $R_{\Delta_{t} \Delta \tau}$ is the cross-correlation function of $\Delta t$ and $\Delta \tau$.

The maximum value of the cross-correlation criterion function for independent $\Delta t$ and $\tau$ is

$\operatorname{Max}_{k=k_{0}}\left\{e_{c c}(k)\right\}=\operatorname{Var}\{\Delta t\}+[E\{\Delta t\}]^{2}$

because $E\{\Delta t\} \neq 0$ here, it is convenient to work with the crosscovariance form of the criterion function

$e_{c o v}(k)=e_{c c}(k)-[E\{\Delta t\}]^{2}$,

then

$\operatorname{Max}_{k=k_{0}}\left\{e_{\operatorname{cov}}(k)\right\}=\operatorname{Var}\{\Delta t\}$.

The quadratic delay difference function $e_{q d d}$ can be presented in the form

$$
\begin{gathered}
e_{q d d}(k)=E\left\{\left\langle|\Delta T(i+k)-\Delta t(i)|^{2}\right\rangle\right\} \\
=2[\operatorname{Var}\{\Delta t\}+\operatorname{Var}\{\tau\}]
\end{gathered}
$$

and the minimum value of the criterion function is

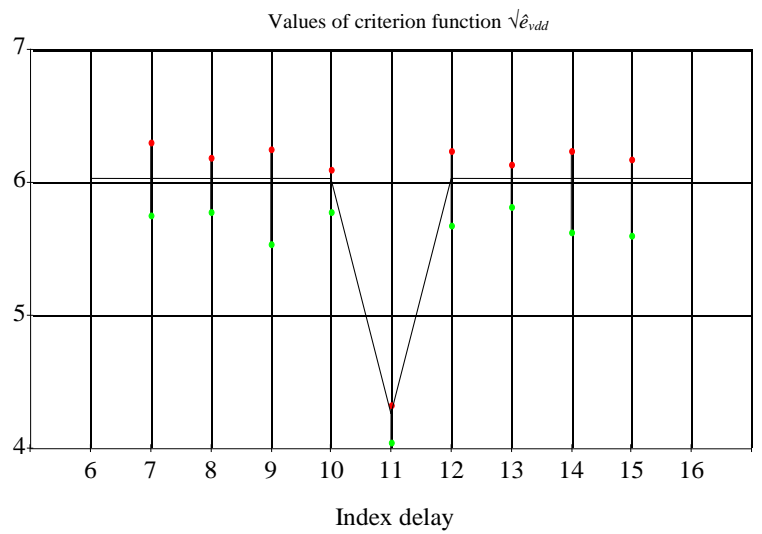

Figure 4. Theoretical and sample values of the square root of $e_{v d d}$ with the same statistics as in Figure 2. Theoretical values computed from the equations (14) and (15) are combined with line. Bar denotes a variation range of sample values in thirty experiments.
Values of criterion function $\hat{e}_{c o r}$

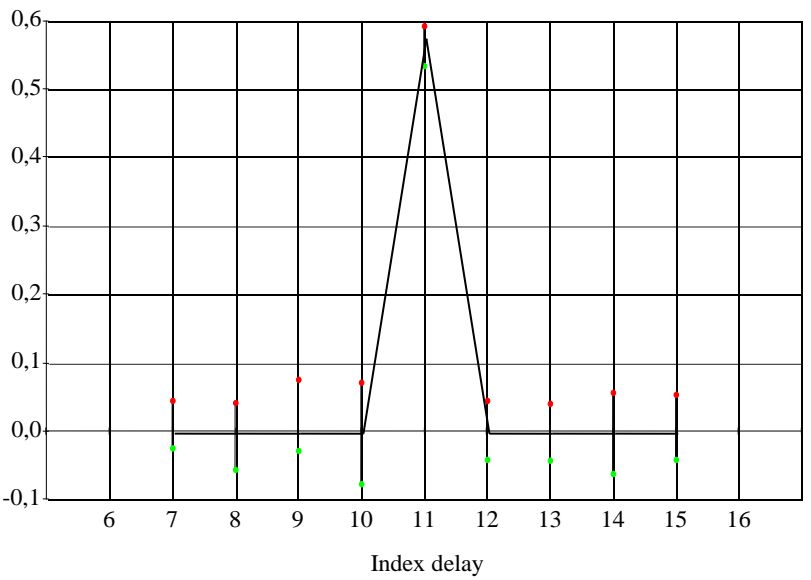

Figure 5. Theoretical and sample values of the criterion function $e_{\text {cor }}$ with the same statistics as in Figure 2. Theoretical values computed from equation (16) and equation (17) are combined with the line. The bar denotes the variation range of the sample values in 30 experiments.

$\operatorname{Min}_{k=k_{0}}\left\{e_{q d d}(k)\right\}=2 \operatorname{Var}\{\tau\}$

if $\Delta t$ and $\tau$ are independent.

Because the maximisation of $e_{c o r}, e_{c c}$, and $e_{c o v}$ is equivalent to the minimisation of $e_{\text {qdd }}$ in stationary systems, Figure 5 only presents the theoretical and sample values of the criterion function $e_{c o r}$.

Theoretical values of the previous criterion functions experimentally approached by the sample criterion functions were derived, assuming stationary stochastic processes generating independent time differences and FIFO-type order relation.

\section{PERFORMANCE OF TIME DIFFERENCE DISTRIBUTION METHOD}

\subsection{Probability of occurrence of an anomalous estimate}

Predicting the performance of time delay estimation methods, it is often possible to set a measure of performance by establishing bounds on performance. For the time delay estimation problem, the Cramer-Rao Lower Bound (CRLB) is commonly used as a performance standard. The CRLB yields a lower bound on the variance of any unbiased time delay estimate as a function of the signal and noise power spectra and the coherent processing time [3], [14]-[16].

Concerning cases of practical interest, there is a theorem that provides that the maximum likelihood estimate can be made arbitrary, close to the CRLB, for sufficiently long observation times [17]. Often, it is not possible to obtain observation times that are sufficiently long. Then, random variations in the values of the criterion function can mask correct extreme values, and large estimation errors or so-called anomalous estimates may occur. Therefore, the actual performance is much worse than the CRLB predicted by the linear analysis for a given observation time and signal-to-noise ratio [18], [19].

Let us estimate the reliability of the time delay estimate of this TDD method, with a finite observation time. The maximum type of the criterion function probability of the occurrence of an anomaly can be described as 
$P_{a}=\int_{-\infty}^{\infty} f_{\hat{e}(m)}(x)\left[1-\prod_{\substack{\left.j=-\frac{\omega}{2} \\ j \neq 0\right)}}^{\frac{\omega}{2}} \int_{-\infty}^{x} f_{\hat{e}(j+m)}(y) d y\right] d x$

where $f_{e}(m)(x)$ and $f_{e(j+m)}(y)$ denote the density functions of independent random variables $\hat{e}(m)$ possessing correct index delay, and $\hat{e}(j+m)$ possessing incorrect index delay if $j \neq 0$. The range of criterion functions (delay windows) over which the extreme value is searched is $\omega+1$ ( $\omega$ is an even number).

The probability of occurrence of an anomaly with the maximum criterion function type with identical Gaussian densities $f_{\hat{e}(k)}(y)$ is in the normalised form

$P_{a}=1-\frac{1}{\sqrt{2 \pi}} \int_{-\infty}^{\infty} e^{-\frac{\theta^{2}}{2}}\left\{\frac{1}{\sqrt{2 \pi}} \int_{-\infty}^{z(\theta)} e^{-\frac{t^{2}}{2}} d t\right\}^{\omega} d \theta$

and

$z(\theta)=\frac{\theta \sqrt{\operatorname{Var}\{\hat{e}(m)\}}+E\{\hat{e}(m)\}-E\{\hat{e}(k)\}}{\sqrt{\operatorname{Var}\{\hat{e}(k)\}}}$

where $k=j+m \neq m$.

Assumption of a Gaussian distribution of $\hat{e}(\cdot)$ is reasonable according to the central limit theorem in view of the large number of random variables summed to produce criterion function $\hat{e}(\cdot)$. Thus, the quantities needed for densities of $\hat{e}(\cdot)$ are their means $E\{\hat{e}(\cdot)\}$ and variances $\operatorname{Var}\{\hat{e}(\cdot)\}$. Given the means and variances of the sample criterion function, the probability of occurrence of an anomaly can be numerically evaluated.

As a special example of the maximum criterion function type is the cross-correlation function (or, in fact, the cross-covariance function) because it turns out that whenever the zero mean assumption is unrealistic (here $E\{\Delta t\}=\mu_{\Delta_{t} \neq 0}$ ), it is more convenient to work with covariance functions than with correlation functions. Assuming a Gaussian distribution, and since $\operatorname{Cov} v_{x y}(\cdot)$ is the unbiased estimate of $\operatorname{Cov}_{x y}(\cdot)$ or $E\left\{\operatorname{Cov}_{x y}(\cdot)\right\}=\operatorname{Cov}_{x y}(\cdot)$, the only other quantity needed for the density of $\operatorname{Cov}_{x y}(\cdot)$ is its variance. The variance of the sample cross-correlation function is given by Van Trees [17] as

$$
\begin{aligned}
\operatorname{Var}\left\{\hat{R}_{x y}(s)\right\}^{\infty} & \\
& \approx \frac{1}{n} \sum_{r=-\infty}^{\infty}\left[R_{x x}(r) R_{y y}(r)\right. \\
& \left.+R_{x y}(r+s) R_{y x}(r-s)\right]
\end{aligned}
$$

and the variance of sample cross-covariance function is

$\operatorname{Var}\left\{\operatorname{Co} v_{x y}(s)\right\} \approx \frac{1}{n} \sum_{r=-\infty}^{\infty}\left[\begin{array}{c}\operatorname{Cov}_{x x}(r) \operatorname{Cov}_{y y}(r) \\ +\operatorname{Cov}_{x y}(r+s) \operatorname{Cov}_{y x}(r-s)\end{array}\right](28)$

Now, the simple model considered here for pre-processed discrete-time signals received at two spatially separate sensors is given by

$$
\left\{\begin{array}{c}
x(i)=\Delta t(i) \\
y(i)=\Delta T(i)=\Delta t(i-m)+\Delta \tau(i-m)
\end{array}\right.
$$

and so

$$
\left\{\begin{array}{c}
\operatorname{Cov}_{x x}(r)=\operatorname{Cov}_{\Delta t \Delta t}(r) \\
\operatorname{Cov}_{y y}(r)=\operatorname{Cov}_{\Delta T \Delta T}(r)=\operatorname{Cov}_{\Delta t \Delta t}(r)+\operatorname{Cov}_{\Delta \tau \Delta \tau}(r) \\
\operatorname{Cov}_{x y}(r+s)=\operatorname{Cov}_{\Delta t \Delta T}(r+s)=\operatorname{Cov}_{\Delta t \Delta t}(r+s-m) \\
\operatorname{Cov}_{y x}(r-s)=\operatorname{Cov}_{\Delta T \Delta t}(r-s)=\operatorname{Cov}_{\Delta t \Delta t}(r-s+m)
\end{array}\right.
$$

Substituting equation (30) into equation (28) yields

$$
\begin{aligned}
& \operatorname{Var}\left\{\operatorname{Cô}_{x y}(s)\right\} \\
& \approx \frac{1}{n} \sum_{r=-\infty}^{\infty}\left\{\begin{array}{c}
\operatorname{Cov}_{\Delta t \Delta t}(r)\left[\operatorname{Cov}_{\Delta t \Delta t}(r)+\operatorname{Cov}_{\Delta t \Delta \tau}(r)\right] \\
+\operatorname{Cov}_{\Delta t \Delta t}(r+s-m) \operatorname{Cov}_{\Delta t \Delta t}(r-s+m)
\end{array}\right\} .
\end{aligned}
$$

Suppose that $s=m$

$$
\begin{aligned}
\operatorname{Var}\left\{\operatorname{Cov}_{x y}(s=m)\right\} \\
\quad \approx \frac{1}{n}\left\{\begin{array}{c}
\operatorname{Cov}_{\Delta t \Delta t}(0)\left[\operatorname{Cov}_{\Delta t \Delta t}(0)+\operatorname{Cov}_{\Delta \tau \Delta \tau}(0)\right] \\
+\operatorname{Cov}_{\Delta t \Delta t}(0) \operatorname{Cov}_{\Delta t \Delta t}(0)
\end{array}\right\} \\
\approx \frac{1}{n}[2 \operatorname{Var}\{\Delta t\}+\operatorname{Var}\{\Delta \tau\}] \operatorname{Var}\{\Delta t\}
\end{aligned}
$$

because

$\left\{\begin{array}{l}\operatorname{Cov}_{\Delta t \Delta t}(0)=\operatorname{Var}\{\Delta t\} \\ \operatorname{Cov}_{\Delta \tau \Delta \tau}(0)=\operatorname{Var}\{\Delta \tau\}\end{array}\right.$

Correspondingly, if $s \neq m$,

$\operatorname{Var}\left\{C \hat{o} v_{x y}(s \neq m)\right\}$

$$
\begin{aligned}
& \approx \frac{1}{n} \sum_{r=-\infty}^{\infty}\left\{\begin{array}{c}
\operatorname{Cov}_{\Delta t \Delta t}^{2}(r)+\operatorname{Cov}_{\Delta t \Delta t}(r) \operatorname{Cov}_{\Delta t \Delta t}(r) \\
+\operatorname{Cov}_{\Delta t \Delta t}(r+s-m) \operatorname{Cov}_{\Delta t \Delta t}(r-s+m)
\end{array}\right\} \\
& \approx \frac{1}{n}\left[\operatorname{Cov}_{\Delta t \Delta t}^{2}(0)+\operatorname{Cov}_{\Delta t \Delta t}(0) \operatorname{Cov}_{\Delta \tau \Delta \tau}(0)\right] \\
& \approx \frac{1}{n} \operatorname{Var}\{\Delta t\}[\operatorname{Var}\{\Delta t\}+\operatorname{Var}\{\Delta \tau\}] .
\end{aligned}
$$

According to equation (26) and the previous discussion, we obtain for the cross-covariance criterion function

$$
\begin{aligned}
z(\theta) \approx & \frac{\theta \sqrt{2\left(\frac{\sigma_{\Delta t}}{\sigma_{\Delta \tau}}\right)^{2}+1}+\left(\frac{\sigma_{\Delta t}}{\sigma_{\Delta \tau}}\right) \sqrt{n}}{\sqrt{\left(\frac{\sigma_{\Delta t}}{\sigma_{\Delta \tau}}\right)^{2}+1}} \\
& =\frac{\theta \sqrt{2\left[\left(\frac{\sigma_{\Delta t}}{\sigma_{\tau}}\right)^{2}+1\right]}+\left(\frac{\sigma_{\Delta t}}{\sigma_{\tau}}\right) \sqrt{n}}{\sqrt{\left(\frac{\sigma_{\Delta t}}{\sigma_{\tau}}\right)^{2}+2}}
\end{aligned}
$$

where $\sigma_{\Delta \tau}=\sigma_{\tau} \sqrt{ } 2, \sigma_{\tau}^{2}=\operatorname{Var}\{\tau\}$ and $\sigma_{\Delta t}^{2}=\operatorname{Var}\{\Delta t\}$.

If we compare equation (25) and equation (35) to the results of Chan, Yansouni, and Crozier in [20], we obtain formal equivalence between the signal-to-noise ratio $(\mathrm{SNR})$ in the conventional cross-correlation method and the $\left(\sigma_{\Delta t} / \sigma_{\Delta \tau}\right)$-ratio or the $\left(\sigma_{\Delta_{t}} / \sigma_{\tau}\right)$-ratio in the TDD method.

According to equation (25) and equation (35), $P_{a}$ is constant when $\left(\sigma_{\Delta_{t}} / \sigma_{\Delta \tau}\right) \sqrt{n}$ is constant, and $\left(\sigma_{\Delta t} / \sigma_{\Delta \tau}\right)^{2}<<1$. This means that the distance between two $P_{\mathrm{a}}$ curves in the $\left(\sigma_{\Delta_{t}} / \sigma_{\tau}\right)$-scale with large sample sizes of $n_{1}(\gg 1)$ and $n_{2}(\gg 1)$ is approximately $10 \log \left(n_{2} / n_{1}\right)$ in decibels, as we clearly see in Figure 6, in which theoretical $P_{a}$ curves computed with equation (25) and equation (35), and the corresponding experimental relative frequencies of anomalous estimates are presented. For example, the probability curves with sample sizes of 1000 and 3000 correspond to each other with a $5 \mathrm{~dB}$ shift (approximately) in respect to the $\left(\sigma_{\Delta t} / \sigma_{\tau}\right)$ - 
ratio, as we see in Figure 6. Each relative frequency point in Figure 6 was obtained from a series of 60 simulation experiments with sample sizes of 300,1000 , and 3000 per experiment. This is in good agreement with the theory presented previously.

\subsection{Threshold effect}

It is well known that below a certain signal-to-noise ratio, the estimation variance increases rapidly and strongly compared to the theoretical variance predicted by linear and high signal-to noise ratio analysis. This is due to the threshold effect caused by anomalous estimates. Of course, the CRLB is no more accurate an estimate of performance for time delay estimators when the probability of occurrence of an anomaly is sufficiently increased from zero. Several studies have been conducted to find a bound that is tighter than the CRLB and that would predict performance more accurately in the case of small signal-to-noise ratios [18]-[23]. For example, it has been evidenced that the ZivZakai Lower Bound (ZZLB) developed in [21] is a tighter bound than CRLB for time delay estimators. In fact, ZZLB is very nearly the greatest lower bound for the dual-sensor delay estimation problem [22].

Generally, the variance in the time delay estimate is characterised by three distinct regions [24]. If the probability of occurrence of an anomaly in the TDD method is increased and approaches $\omega /(\omega+1)$, prior information (e.g. the known maximum delay) limits the variance. This is equivalent to a pure guess of the index delay in the selected delay window. Obviously, then, we can simply assume a uniform distribution of anomalies between the limits of the delay window as Ianniello does in [18]. If the probability of occurrence of an anomaly approaches zero, the variance of the time delay estimate approaches CRLB. Between these limits, there is a transition region from the prior information limit to the linear mode or CRLB. The theoretical variance of the time delay estimate in the presence of anomalous estimates can be determined from Ianniello's correlator performance estimate (CPE) model [18], which is modified here to

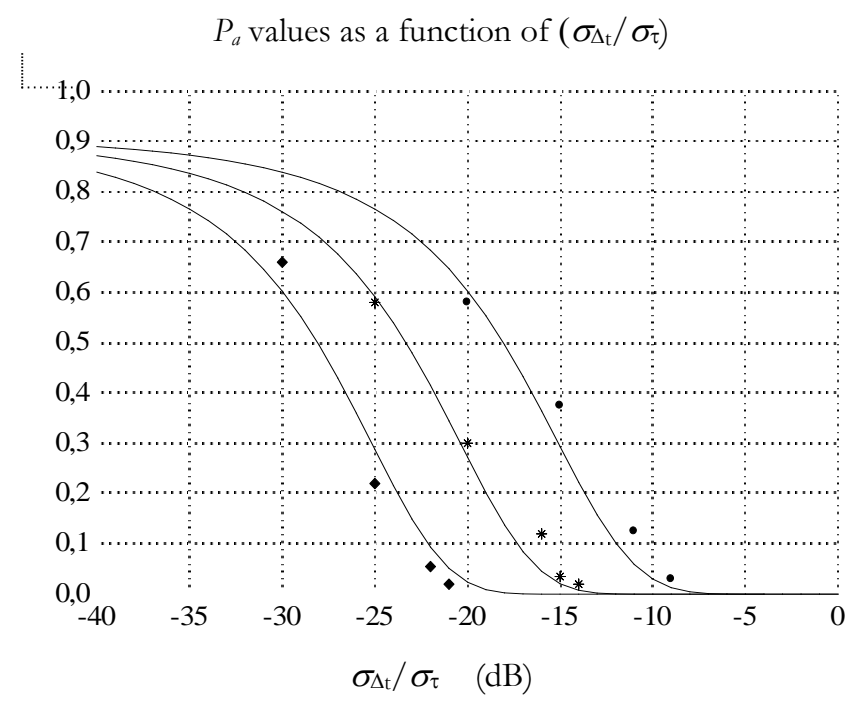

Figure 6. Probability curves and corresponding relative frequencies of occurrence of an anomalous estimate with $\omega=10$ and sample sizes 300 (• • $\bullet), 1000(* * *)$ and $3000(\bullet \bullet)$ samples. Each relative frequency point was obtained from series of sixty simulation experiments with sample sizes of 300,1000 and 3000 per experiment.
$\operatorname{Var}\{\langle\tau\rangle\}=\left(1-P_{a}\right) \operatorname{Var}\{\langle\tau\rangle\}_{\min }+P_{a} \frac{[(\omega+1) E\{\Delta t\}]^{2}}{12}$

where $\operatorname{Var}\{\langle\tau\rangle\}_{\text {min }}$ is a variance of sample mean $\langle\tau\rangle$ below the threshold.

In normalised form, the variance of $\langle\tau\rangle$ is

$\operatorname{Var}\{<\tau\rangle\}_{\text {norm }}=\frac{\left.\left(1-P_{a}\right) \operatorname{Var}\{<\tau\rangle\right\}_{\min }}{\frac{[(\omega+1) E\{\Delta t\}]^{2}}{12}}+P_{a}$.

An interval between two $\operatorname{Var}\{\langle\tau\rangle\}_{\text {norm }}$ curves in $\left(\sigma_{\Delta_{t}} / \sigma_{\tau}\right)$ scale with large sample sizes of $n_{1}$ and $n_{2}$ is (approximately) $10 \log \left(n_{2} / n_{1}\right)$ in decibels. According to the previous discussion of constant $P_{a}$ values, $P_{a}$-curves intersect the corresponding (same sample size) $\operatorname{Var}\{\langle\tau\rangle\}_{\text {min }}$ curves (linear parts of Figure 7) with an approximately constant value of normalised $\operatorname{Var}\{<\tau>\}$ as long as $\left(\sigma_{\Delta t} / \sigma_{\Delta \tau}\right)^{2}<<1$ or $\left(\sigma_{\Delta t} / \sigma_{\tau}\right)^{2}<<2$, which necessarily means that the sample size is large because the constant threshold value of $P_{\mathrm{a}}$ is much smaller than one.

Thus, the threshold variance, or threshold time delay error, is approximately constant independent of the sample size. The threshold $\left(\sigma_{\Delta t} / \sigma_{\Delta \tau}\right)$-ratio, at which the variance of the time delay estimate begins to deviate from the minimum variance corresponding to CRLB, is approximately proportional to $1 / \sqrt{n}$ or to $-10 \log (n)$ in decibels. These results are equivalent to the results of Scarborough, Tremblay, and Carter [24], [25]. They observed that the value of variance at which ZZLB begins to deviate from CRLB remains essentially constant, independent of the coherent processing time, with a large bandwidth-time product. The threshold signal-to-noise ratio is approximately inversely proportional to the square root of the coherent processing time for the large bandwidth-time product.

Figure 7 demonstrates the sample sizes of 300, 1000, 3000, and 10000 for which the theoretical threshold variances stay essentially constant with large sample sizes. Figure 7 shows that a sample size of 100 samples is not sufficiently large. The threshold value condition $\left(\sigma_{\Delta_{t}} / \sigma_{\tau}\right)^{2}<<2$ is strongly violated. Sample variances and theoretical variances of $\langle\tau\rangle$ correspond quite well. Only the experimental threshold $\left(\sigma_{\Delta t} / \sigma_{\tau}\right)$-ratio seems to be decreased in the order of $5 \mathrm{~dB}$ compared to the theoretical threshold. Clearly, this effect is caused by the small number of experiments. For example, the probability that at least one anomalous estimate occurs in the nearest neighbourhood of the threshold $\left(\sim 10^{-6}\right.$ in Figure 7$)$ with 60 experiments is 0.00006 assuming the experiment outcome 'anomalous estimate' follows the binomial distribution.

The threshold effect has particular significance concerning coherent vs. incoherent signal processing for time delay estimation. Incoherent processing is an attractive alternative to coherent processing in many time delay applications of conventional correlation techniques because it becomes necessary to compensate for the effect of variations in time delay (e.g. due time-varying time delay, source or receiver motion) for coherent processing times other than short ones [26], [27], [28].

In incoherent processing, the whole sample size is divided into sections. The sections are processed individually to obtain one estimate for each section. These time delay estimates are then averaged to obtain the final estimate at the end of the whole observation interval. The coherent processing sample size with incoherent processing is reduced to $n / N$ samples for each of the 


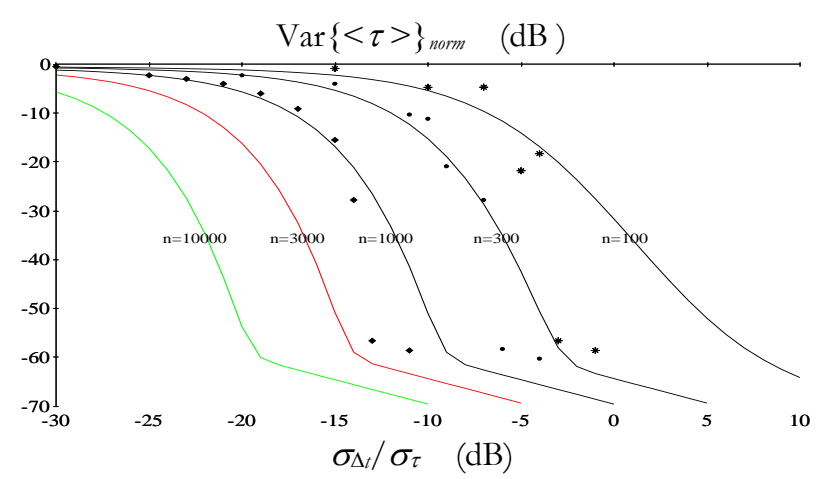

Figure 7. Normalised theoretical and sample variances of $\langle\tau\rangle$ as a function of the $\left(\sigma_{\Delta t} / \sigma_{\tau}\right)$-ratio. The sample size is the parameter. The theoretical variance curves are numerically computed using equation (25), equation (35), and equation (37) with $\omega=10, \mu_{\Delta t}=30$, and $\sigma_{\Delta t}=1$. Each sample variance is calculated from 60 simulation experiments using sample sizes $n=100(* * *)$, $n=300(\bullet \bullet)$, and $n=1000(\bullet \bullet \bullet)$.

$N$ sections, although the total observation sample size is still $n$ samples.

If the actual $\left(\sigma_{\Delta t} / \sigma_{\tau}\right)$-ratio is larger than the $\left(\sigma_{\Delta t} / \sigma_{\tau}\right)_{n / N^{-}}$-ratio, which is the threshold $\left(\sigma_{\Delta_{t}} / \sigma_{\tau}\right)$-ratio for the coherent processing sample size of $n / N$ samples, the performance of the incoherent processor (according to previous threshold discussions) coincides with the coherent processor and with small error performance. However, if

$\left(\frac{\sigma_{\Delta t}}{\sigma_{\tau}}\right)_{n}<\left(\frac{\sigma_{\Delta t}}{\sigma_{\tau}}\right)<\left(\frac{\sigma_{\Delta t}}{\sigma_{\tau}}\right)_{\frac{n}{N}}$

the incoherent processor exhibits significantly poorer performance than the coherent processor. If $n / N$ is large, the threshold $\left(\sigma_{\Delta t} / \sigma_{\tau}\right)$-ratio for the coherent processing sample size of $n / N$ samples is

$\left(\frac{\sigma_{\Delta t}}{\sigma_{\tau}}\right)_{\frac{n}{N}} \approx\left(\frac{\sigma_{\Delta t}}{\sigma_{\tau}}\right)_{n} \sqrt{N}$.

Thus, significant performance gains could be obtained for small $\left(\sigma_{\Delta t} / \sigma_{\tau}\right)$-ratios by increasing the coherent processing time. Note that the analogy between the $\left(\sigma_{\Delta_{t}} / \sigma_{\tau}\right)$-ratio in TDD and the signal-to-noise ratio in GCC continues, as we can see that comparing equation (39) to the following equation holds for large bandwidth-time products [24]

$S N R_{\bar{N}} \approx 5 \log (N)+S N R_{T}$

where $S N R_{\mathcal{T}}$ is the threshold $S N R$ for a coherent processing time of $\mathcal{T}$, and $S N R_{\mathcal{T} / N}$ is the threshold $S N R$ for a coherent processing time of $\mathcal{T} / N$.

In the TDD method, it is not necessary to compensate for the effect of moderate time variations in the time delay because the constant index delay giving the extreme value to the criterion function also contains information on the variable time delay and determines the best estimate for time delay distribution within the meaning of that criterion. In the conventional correlation method with coherent processing, there is only a constant time delay argument, which maximises the cross-correlation function without noticing a variable time delay. Of course, if the time delay is strongly and constantly changing, it may destroy a sufficient statistical similarity (correlation) between the time sequences in TDD with coherent processing so that only random noise and sensor error effects remain. Thus, the cessation of the error propagation coherent processing time in TDD is also bound. Then, a prerequisite for the successful utilisation of incoherent processing is the condition $\left(\sigma_{\Delta_{t}} / \sigma_{\tau}\right)>\left(\sigma_{\Delta_{t}} / \sigma_{\tau}\right)_{n / N}$.

\subsection{Distortion of time delay distribution in flow systems disobeying the FIFO order relation}

The theoretical values of the previous criterion functions were derived assuming stationary stochastic processes generating independent time differences and FIFO-type order relations between sensors.

If the flow system is not a FIFO system, time delay distribution, coherently computed with the help of information on two time sequences obtained from two sensors a fixed distance apart, is a distorted version of true time delay distribution. Such a situation may, for example, be in traffic applications (a multilane motorway with unlimited speed as a pathological example) where the distance between the sensors is sufficiently long that this relation $\mu_{\Delta_{t}}<<\mu_{\tau}$ is true and the delay time distribution of the vehicles is sufficiently wide that this relation $\sigma_{\tau}<<\mu_{\Delta t}$ is not true, the consequence being that the time delay distribution detected by the measurement system is distorted (without identification of the vehicles and their arrival and departure times).

The results of applying the theory presented in the previous sections are fully applicable if the distortion is small. Obviously, the distortion is small if the following expression

$P_{0}=\int_{-\infty}^{\infty} f_{\Delta t}(\Delta t) \int_{-\Delta t}^{\Delta t} f_{\Delta \tau}(\Delta \tau) d \Delta \tau d \Delta t$

where

$f_{\Delta \tau}(\Delta \tau)=\int_{-\infty}^{\infty} f_{\tau}(\tau) f_{\tau}(\Delta \tau-\tau) d \tau$

is near to one. $P_{0}$ describes the probability per sample that the FIFO order relation is not violated. Quite arbitrary, we could require that the condition of the small distortion is valid if $P_{0}$ is in the order of 0.9 or more.

To obtain a simple approximation, we replace $f_{\Delta_{t}}$ with Dirac's delta function $\delta_{D}(\cdot)$

$f_{\Delta t}(\Delta t)=\delta_{D}(\Delta t-E\{\Delta t\})$.

Then

$P_{0}=F_{\Delta \tau}(E\{\Delta t\})-F_{\Delta \tau}(-E\{\Delta t\})$

where $F_{\Delta \tau}(\cdot)$ is a cumulative distribution function of random variable $\Delta \tau$. Assuming Gaussian variable $\tau$, equation (43) becomes the simple form

$P_{0}=2 \operatorname{erf}\left(\frac{\mu_{\Delta t}}{\sigma_{\tau} \sqrt{2}}\right)$

where $\operatorname{erf}(\cdot)$ is the error function

$\operatorname{erf}(x)=\frac{1}{\sqrt{2 \pi}} \int_{0}^{x} e^{-\frac{y^{2}}{2}} d y$.

Now, the condition of the previously determined small distortion holds if $\mu_{\Delta_{t}} / \sigma_{\tau} \geq 2.33$.

For Gaussian variables $\Delta t$ and $\tau$,

$P_{0}=2 \operatorname{erf}\left(\frac{\mu_{\Delta t}}{\sqrt{\sigma_{\Delta t}^{2}+2 \sigma_{\tau}^{2}}}\right)$ 


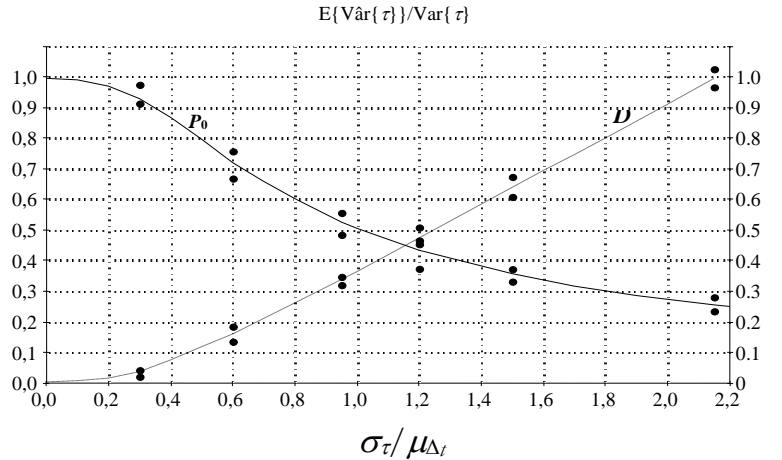

Figure 8. Reduction of the detected variance of transport delay and the distortion of delay distribution with Gaussian variables $\left(\mu_{\Delta t}=30, \sigma_{\Delta t}=5\right)$. Each sample variance is computed from 1000 samples. Theoretical curves are computed from equation (45), equation (47), and equation (48). Black spots denote the variation range of the sample values in 20 simulation experiments.

and the condition of small distortion holds if

$\frac{\mu_{\Delta t}}{\sqrt{\sigma_{\Delta t}^{2}+2 \sigma_{\tau}^{2}}} \geq 1.65$.

As a measure of distortion, we could use the following expression

$D=\sum_{i=1}^{\infty} i \int_{-\infty}^{\infty} f_{\Delta t}(\Delta t) \int_{-(i+1) \Delta t}^{-i \Delta t} f_{\Delta \tau}(\Delta \tau) d \Delta \tau d \Delta t$.

$D$ describes the average amount of FIFO order violations per sample. For Gaussian variables $\Delta t$ and $\tau$, equation (46) can be obtained after manipulation to form

$D=\sum_{i=1}^{\infty} i\{\operatorname{erf}[A(i+1)]-\operatorname{erf}[A(i)]\}$

where

$A(i)=\frac{i \mu_{\Delta t}}{\sqrt{i \sigma_{\Delta t}^{2}+2 \sigma_{\tau}^{2}}}$.

In Figure 8, we see that the condition for the small distortion holds if the distortion percentage $(100 \% \cdot D)$ is about $5 \%$ or smaller.

The expectation of the sample variance of the estimated time delay is reduced due to distortion

$E\{\operatorname{Var}\{\tau\}\}=P_{0} \operatorname{Var}\{\tau\}$

where $P_{0}$ is obtained from equation (41). Therefore, the previous condition for the small distortion means that the standard deviation of the time delay is underestimated at approximately 5 $\%$ or less if the idealised FIFO model is used. In Figure 8 , the reduction of $E\{\operatorname{Var}\{\tau\}\}$ from its true value $\operatorname{Var}\{\tau\}$ with Gaussian variables $\Delta t$ and $\tau$ as a function of $\sigma_{\tau} / \mu_{\Delta t}$ presents holding $\sigma_{\Delta t}$ as a constant. Figure 8 shows rather good harmony between the experimental results and the theoretical curves computed from equation (45), equation (47), and equation (48). Each sample variance and distortion percentage were computed from the experiment of 1000 time samples.
Of course, the reduced sample variance of the time delay due to distortion will bias the estimate of mean transport velocity. However, the estimate of mean transport delay is still unbiased because of the compensation mechanism due to the inherent symmetry of distortion effects. However, violation of the FIFO order or mixing in the space between the sensors effectively reduces the sufficient statistical similarity (correlation) between the channel signals, which is an essential prerequisite for the successful utilisation of the method. Therefore, a sufficient statistical similarity between channel signals sets such an upper limit for the distance between sensors that the previous condition for the small distortion cannot disobey much.

\subsection{Some discussions on reliability-checking possibilities}

Because the TDD method is based on the utilisation of criterion functions, there are several simple ways, in smart measurement systems, of implementing automatic reliability checking or supervisory operations even in real time. The trivial checking operation is based on magnitude testing of the extreme value of the criterion function. If the extreme value exceeds or passes under some predetermined range, it is reasonable to doubt the reliability of results. Of course, a great disadvantage of this simple checking method is the determination of the acceptable range of extreme values, which may be problematic.

Obviously, it is possible to utilise the theoretical relationship between the extreme value of the criterion function and the $\left(\sigma_{\Delta t} / \sigma_{\tau}\right)$-ratio to fix the predetermined range. For example, choosing the lowest acceptable limit of $\left(\sigma_{\Delta t} / \sigma_{\tau}\right)$, we get the lowest acceptable maximum value of the criterion function and vice versa. Fixing the sample size and index delay window, we get a clear probability measure $P_{a}$ for a criterion of reliability. For example, the probability of an anomalous estimate as a function of the maximum value of the normalised cross-covariance criterion function $e_{c o r}$ is

$P_{a}=1-\frac{1}{\sqrt{2 \pi}} \int_{-\infty}^{\infty} e^{-\frac{\theta^{2}}{2}}\left\{\frac{1}{\sqrt{2 \pi}} \int_{-\infty}^{z(\theta)} e^{-\frac{t^{2}}{2}} d t\right\}^{\omega} d \theta$,
$Z(\theta)=\theta \sqrt{\operatorname{Max}_{k}\left\{e_{c o r}(k)\right\}^{2}+1}+\sqrt{n} \operatorname{Max}_{k}\left\{e_{c o r}(k)\right\}$.

Figure 9 shows several $P_{\mathrm{a}}$-curves with some sample sizes.

The second possibility concerning the evaluation unreliability of measurement results is to study the sensitivity of the criterion function in surroundings of selected index delay. Obviously, we can think that a result is reliable if the extreme value differs sufficiently from nearby values. Determination of a sufficient difference between the extreme value and other values of the criterion function is also problematic. A quantitative measure of the quality of the time delay estimator in the delay window of $(\omega+1)$ index values ( $\omega$ is even) could be

$$
\hat{u}=\frac{\hat{e}\left(k_{0}\right)-\frac{1}{\omega} \sum_{|i|=1}^{\frac{\omega}{2}} \hat{e}\left(k_{0}+i\right)}{\sqrt{\frac{1}{\omega} \sum_{|i|=1}^{\frac{\omega}{2}}\left[\hat{e}\left(k_{0}+i\right)-\frac{1}{\omega} \sum_{|i|=1}^{\frac{\omega}{2}} \hat{e}\left(k_{0}+i\right)\right]^{2}}}
$$

where $k_{0}$ is the estimated index delay. A similar quality measure ('peakedness') was presented by Mars and Van Arragon using the concept of the average mutual amount of information in the estimation of time delay in nonlinear systems [29].

We can further expand this idea. For example, in the case of the cross-covariance criterion function, a comparison of 


\section{$P_{a}(\mathrm{~dB}$ below 1)}

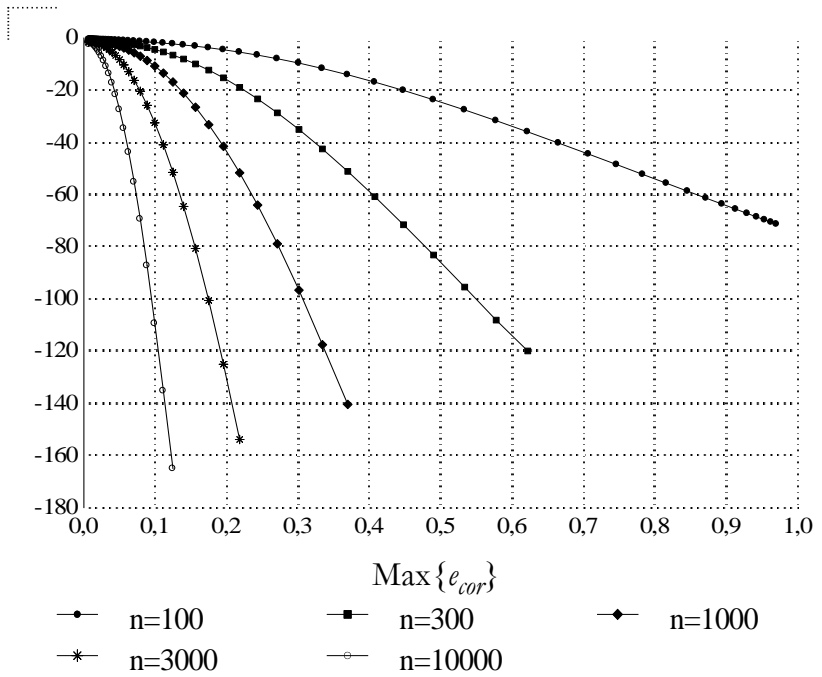

Figure 9. The probability of an anomalous estimate as a function of the maximum value of normalised cross-covariance criterion function computed by equation (49), and $\omega=10$.

equation (26), equation (35), and equation (50) shows that measure $\hat{u}$ is an estimate of

$u=\frac{E\left\{\hat{e}\left(k_{0}\right)\right\}-E\{\hat{e}(k)\}}{\sqrt{\operatorname{Var}\{\hat{e}(k)\}}} \approx \frac{\sqrt{n}\left(\frac{\sigma_{\Delta t}}{\sigma_{\tau}}\right)}{\sqrt{\left(\frac{\sigma_{\Delta t}}{\sigma_{\tau}}\right)^{2}+2}}$

where $k \neq k_{0}$.

In this way, we can transform this measure of 'peakedness' to the probability of occurrence of an anomaly, substituting

$z(\theta)=\theta \sqrt{\frac{u^{2}}{n}+1}+u$

into equation (49).

The third possibility concerning supervision of reliability is more practical and is ready to use. This method utilises several different criterion functions at the same time. An agreement of criterion functions concerning index delay is the measure of reliability. The advantage of this method is that it is not necessary to determine any threshold values or ranges.

Simultaneous use of several different criterion functions in reliability checking is based on the presumption that it is very

Table 1. The relative amount of undetected errors (anomalous estimates) as a function of $\left(\sigma_{\Delta t} / \sigma_{\tau}\right)$-ratio and corresponding reference probability of an anomalous estimate with cross-covariance criterion function $P_{a}$ cov. All distributions are approximately Gaussian. Each figure describing the relative amount of undetected error of criterion functions $\left(e_{c o v}, e_{\text {add }}, e_{v d}\right)$ is calculated from 60 experiments.

\begin{tabular}{c|c|c|c|c|c|c|c}
\hline \multicolumn{2}{c|}{$\begin{array}{l}\sigma_{\Delta t} / \sigma_{\tau} \\
\text { in dB }\end{array}$} & \multicolumn{2}{c|}{$P_{a}{ }^{c o v}$} & \multicolumn{3}{c}{$\begin{array}{c}\text { Relative amount of undetected errors } \\
e_{\text {cov }} \text { only }\end{array}$} & \multicolumn{2}{c}{$\begin{array}{c}\text { Combination of } \\
e_{\text {add }}, e_{c o v} \text { and } e_{v d}\end{array}$} \\
\hline$n=100$ & $n=300$ & $n=300$ & $\omega=10$ & $n=100$ & $n=300$ & $n=100$ & $n=300$ \\
\hline 0 & -5 & $\ll 0.001$ & 0 & 0 & 0 & 0 \\
\hline-5 & -10 & 0.030 & 0.02 & 0.05 & 0 & 0 \\
\hline-10 & -15 & 0.296 & 0.27 & 0.38 & 0.02 & 0.07 \\
\hline-15 & -20 & 0.606 & 0.57 & 0.62 & 0.13 & 0.05 \\
\hline-20 & -25 & 0.769 & 0.73 & 0.72 & 0.08 & 0.10 \\
\hline-25 & -30 & 0.841 & 0.87 & 0.85 & 0.10 & 0.08 \\
\hline
\end{tabular}

unlikely that all different criterion functions have the extreme value in the same incorrect index delay. This presumption seems to be correct, as we see in Table 1, in which each figure describes the relative number of undetected errors calculated in 60 experiments. Due to the error detection capability of combined criterion functions, the relative amount of the undetected error strongly decreases compared to the single reference criterion function (the main criterion function), which in Table 1 , is a cross-covariance function $\hat{e}_{c o v}$.

The fourth possible method for the reliability evaluation of results is also practical and ready to use in smart measurement systems. This method utilises coherent and incoherent processing at the same time, continually comparing the magnitudes of extreme values and detecting optimum index delay changes, which may reveal errors caused by strongly and constantly changing time delays or all types of sensor errors, which may destroy a sufficient statistical similarity (correlation) between time sequences. An agreement on the optimum index delay is the measure of reliability. The advantage of this method is that it is not necessary to determine any threshold values or ranges.

\section{CONCLUSION}

In this time delay estimation model, the criterion function compares the time differences of time sequences between channels, not the amplitudes of time functions as in the conventional cross-correlation method. Using the index delay, which gives the extreme value to the criterion function, it is possible to calculate the best estimate for time delay distribution within the meaning of that criterion. With the help of estimated time delay distribution, the velocity distribution can be calculated when the distance between sensors is known. Using this method, the estimated delay distribution and criterion function are clearly separated. There is no need to make symmetry assumptions for the criterion function or to assume the criterion function as an estimate of the time delay distribution. Thus, there are no theoretical problems in the determination of average time delay or velocity in the non-constant or changing time delay case as long as a sufficient statistical similarity (correlation) exists between the channel signals.

The theoretical values of several criterion functions and the probability of occurrence of an anomalous estimate with the cross-covariance criterion function are derived. The threshold effect caused by anomalous estimates, coherent vs. incoherent processing, and the distortion of estimated delay distribution are discussed. Some possibilities concerning reliability supervision based on the use of criterion functions are outlined. Potential applications of the time difference distribution method are the delay and velocity measurements in stochastic flow, or the transport processes and time delay estimation problems in radar, sonar, and wireless communication systems.

The basis and an analysis of the estimation method using simple data were presented here. Topics for additional studies could be, for example, favourable subjects of practical application and real-time reliability-checking methods based on simultaneous use of different criterion functions and parallel incoherent and coherent processing in smart measurement systems.

\section{REFERENCES}

[1] G. C. Carter, Special issue on time delay estimation, IEEE Trans. Acoust., Speech, Signal Processing ASSP-29, 3 (1981). 
[2] J. S. Bendat, A. G. Piersol, Engineering Applications of Correlation and Spectral Analysis, Wiley-Interscience, New York, 1980.

[3] C. H. Knapp, G. C. Carter, The generalized correlation method for estimation of time delay, IEEE Trans. Acoust., Speech, Signal Processing ASSP-24, 4 (1976) pp. 320-327.

[4] M. H. Butterfield, G. F. Bryant, J. Dowsing, A new method of strip speed measurement using random waveform correlation, Trans. Soc. Instrum. Tech. 13 (1961) pp. 111-123.

[5] T. Idowaga, T. Ono, A new method of vehicle-speed measurement using cross correlation, Proc. of the VII IMEKO World Congress, London, UK, 10 - 14 May 1976, Institute of Measurement and Control, London pp. BLV 218/1-9.

[6] C. Zimmer, H. Ryser, H. Meyr, An instrument for non contact speed measurement by image correlation, Proc. of the VII IMEKO World Congress, London, UK, 10 - 14 May 1976, Institute of Measurement and Control, London, pp. BLV 217/110.

[7] H. Kashiwagi, Paper strip speed measurement using correlation techniques, Trans. Soc. Instrument and Contr. Engrs., Japan, 1968, pp. 4304-312.

[8] M. S. Beck, Correlation in instruments: cross correlation flowmeters, J. Phys. E: Sci. Instrum. 14 (1981) pp. 7-19.

[9] J. W. Arthur, J. F. Dix, E. Harland, J. W. Widdowson, P. B. Denyer, J. Mavor, A. D. Milne, Large time-bandwidth product CCD correlators for sonar, IEE Conference Publication 1979/180, pp. 62-68.

[10] D. E. N. Davies, Signal processing for radar applications, IEE Conference Publication no. 1979/180, pp. 145-150.

[11] W. H. Haas, C. S. Lindquist, A synthesis of frequency domain filters for time delay estimation, IEEE Trans. Acoust., Speech, Signal Processing ASSP-29 (1981) pp. 540-548.

[12] R. M. Henry, An improved algorithm allowing fast on-line polarity correlation by microprocessor or minicomputer, IEEE Colloquium on Correlation Processing, May 1974, London, UK pp. 3/1-4.

[13] L. R. Morris, The role of zero crossings in speech recognition and processing, Proc. of the IEEE Conf. Speech Comm. Processing, Boston, USA, 24 - 26 April 1972, pp. 446-450.

[14] B. Friedlander, On the Cramer-Rao bound for time delay and Doppler estimation, IEEE Trans. Inform. Theory, vol. IT-30, May 1984, pp. 575-580.

[15] W. R. Hahn, Optimum signal processing for passive sonar range and bearing estimation, J. Acoust. Soc. Amer. 58 (1975) pp. 201 207.

[16] R. L. Kirlin, J. N. Bradley, Delay estimation simulations and a normalized comparison of published results, IEEE Trans. Acoust., Speech, Signal Processing ASSP-30 (1982) pp. 508-511.

[17] H. L. Van Trees, Detection, Estimation, and Modulation Theory, Part I, John Wiley and Sons, New York, 1968.
[18] J. P. Ianniello, Time delay estimation via cross-correlation in the presence of large estimation errors, IEEE Trans. Acoust., Speech, Signal Processing ASSP-30 (1982) pp. 998-1003.

[19] J. P. Ianniello, Large and small error performance limits for multipath time delay estimation, IEEE Trans. Acoust., Speech, Signal Processing ASSP-34 (1986) pp. 245-251.

[20] Y. T. Chan, P. Yansouni, S. Crozier, Time delay estimation with low signal to noise ratios, Proc. of the IEEE International Conference on Acoustics, Speech, and Signal Processing ICASSP '84, 19 - 21 March 1984, San Diego, USA, pp. 616-619.

[21] A. Weiss, E. Weinstein, Fundamental limitations in passive time delay estimation - Part I: Narrow-band systems, IEEE Trans. Acoust., Speech, Signal Processing ASSP-31 (1983) pp. 472-486.

[22] J. P. Ianniello, E. Weinstein, A. Weiss, Comparison of the ZivZakai lower bound on time delay estimation with correlator performance, Proc. of the IEEE International Conference on Acoustics, Speech, and Signal Processing ICASSP '83, Boston, USA, 14-16 April 1983, pp. 875-878.

[23] J. P. Ianniello, Comparison of the Ziv-Zakai lower bound on multipath time delay estimation with autocorrelator performance, ICASSP 84, Proc. of the IEEE International Conference on Acoustics, Speech, and Signal Processing ICASSP '84, 19 - 21 March 1984, San Diego, USA, pp. 620-623.

[24] K. Scarborough, R. J. Tremblay, G. C. Carter, Performance predictions for coherent and incoherent processing techniques of time delay estimation, IEEE Trans. Acoust., Speech, Signal Processing ASSP-31 (1983) pp. 1191-1196.

[25] K. Scarborough, G. C. Carter, R. J. Tremblay, Implications of threshold effects for coherent and incoherent processing techniques of time delay estimation, Proc. of the IEEE International Conference on Acoustics, Speech, and Signal Processing ICASSP ‘84, 19 - 21 March 1984, San Diego, USA, pp. 612-615.

[26] C. H. Knapp, G. C. Carter, Estimation of time delay in the presence of source or receiver motion, J. Acoust. Soc. Amer. 61 (1977), pp. 1545-1549.

[27] W. B. Adams, J. P. Kuhn, W. P. Whyland, Correlator compensation requirements for passive time delay estimation with moving source or receivers, IEEE Trans. Acoust., Speech, Signal Processing ASSP-28, 2 (1980), pp. 158-168.

[28] J. P. Kuhn, S. E. Robison, D. W. Winfield, Time Delay Estimation Techniques for Moving Sources, Proc. of the 24th Midwest Symp. on Circuits and Systems, Albuquerque, USA, 29 June 1981, pp. 6975.

[29] N. J. I. Mars, G. W. Van Arragon, Time delay estimation in nonlinear systems, IEEE Trans. Acoust., Speech, Signal Processing ASSP-29 (1981) pp. 619-621. 